\title{
Pain Management of Budd Chiari Syndrome in the Primary Care Setting: A Case Study
}

Pilar Z. Murphy, PharmD, MPH, BCACP; Jimiece Thomas, PharmD \& MPH Candidate; Taylor P. McClelland, PharmD \& MPH Candidate Samford University McWhorter School of Pharmacy

\begin{abstract}
Introduction: Budd Chiari Syndrome (BCS) is a very rare disease affecting approximately 1 in 100,000 people in the general population. It is caused by an obstruction of the hepatic veins leading to blood backing up in the liver. Treatment options to improve hepatic blood flow and relieve ascites are well documented. However, there are no established guidelines or treatment preferences for pain associated with BCS while patients are awaiting other treatment options.

Case: A 22-year-old African American female was diagnosed with Budd Chiari Syndrome. The initial attempt at a transjugular intrahepatic portosystemic shunt (TIPS) procedure failed. While awaiting a second attempt at the procedure, the patient presented to her primary care provider complaining of abdominal and right upper quadrant pain. Treatment guidelines were searched for acute pain management options; however, no BCS pain management guidelines exist.

Discussion: Individuals with BCS often present with abdominal pain, however, no guidelines outlining analgesic options in BCS exists. Acetaminophen, NSAIDs, and opioids are commonly used prescription medications for moderate to severe pain. Acetaminophen use was not considered due to acute liver injury and portal venous thrombosis. Anticoagulation with apixaban prevented concurrent use with NSAIDs. Opioid medications combined with acetaminophen were excluded to minimize exacerbating the liver injury. Tramadol $25 \mathrm{mg}$ was chosen due to its lower abuse profile than other opioid analgesics, and was initiated for pain management.

Conclusion: The patient reported adequate pain control with tramadol, tolerated the medication with no complications, and underwent a successful TIPS procedure one month later. Abdominal pain is a common symptom of BCS and needs to be effectively managed. Guidelines on treating pain associated with BCS in the outpatient setting would improve quality of life for patients and provide guidance to primary care providers requiring direction on how to address pain associated with Budd Chiari Syndrome safely and adequately.
\end{abstract}

Keywords: Budd Chiari Syndrome, pain, primary care, guidelines

\section{Introduction}

Budd Chiari Syndrome (BCS) is a very rare, and not well reported disease that affects approximately 1 in 100,000 people in the general population. ${ }^{1,2}$ The syndrome is caused by a blockage or obstruction of the hepatic veins that leads to blood backing up in the liver, causing liver and possibly spleen enlargement. ${ }^{2}$ Hepatic vein blockade is commonly due to a thrombosis, but could also be due to a non-thrombotic obstruction. The thrombus may extend to also block the inferior vena cava. This blockade leads to portal hypertension and its associated complications such as abdominal ascites. ${ }^{2,3}$ Some patients with BCS may have an initial presentation of acute liver failure. However, the hallmark symptoms of hepatomegaly, ascites, and abdominal pain are present in the majority of patients diagnosed with Budd Chiari Syndrome. ${ }^{2,4}$ If left untreated, the prognosis of BCS is poor, with a survival rate of about $6.8 \%$ if left untreated beyond five years of diagnosis. ${ }^{2}$ Risk factors for BCS include conditions that increase blood clotting-polycythemia and thrombocythemia, sickle cell disease, obesity, and pregnancy as well as Janus tyrosine kinase-2 V617F mutations. ${ }^{5}$ Inherited thrombotic disorders such as Protein $\mathrm{C}$ deficiency, Protein $\mathrm{S}$ deficiency and Factor $\mathrm{V}$ Leiden deficiency may also increase a person's risk of developing BCS. There are other identified causes of this rare

Corresponding author: Pilar Z. Murphy, PharmD, MPH, BCACP Clinical Associate Professor of Pharmacy Practice Samford University McWhorter School of Pharmacy Email: pmurphy@samford.edu disease including pyrrolizidine plant alkaloids, oral contraception use, inflammatory disorders, and Asian or South African ancestry. ${ }^{3}$ Treatment options for BCS to improve hepatic blood flow and relieve ascites are well documented. Conversely, there seem to be no established treatment options for patients with pain associated with BCS while they are awaiting some of the treatment options. This case is noteworthy in that it highlights the need for additional treatment guidelines for pain in patients with Budd Chiari Syndrome.

\section{Case}

A 22-year-old African American female who presented to her primary care provider in the United States rural south, an area with limited access to specialist care. The patient first presented complaining of malaise and fatigue. One month later, she presented complaining of the same symptoms and laboratory monitoring was performed. Abnormal laboratory findings included elevated alkaline phosphatase at $147 \mathrm{IU} / \mathrm{L}$ (normal range: 39-117), AST (SGOT) 58 IU/L (normal range: 040), and ALT (SGPT) of $75 \mathrm{IU} / \mathrm{L}$ (normal range 0-32). Bilirubin $(0.5 \mathrm{mg} / \mathrm{dL}$ ) was normal (normal range: $0.0-1.2)$. At this time, the patient was thought to have acute hepatitis and a urinary tract infection. After about five months, the patient presented back to her primary care provider complaining of abdominal pain, nausea, vomiting, dark urine, decreased appetite, light colored stool, ascites, and jaundiced eyes. Due to the COVID-19 pandemic, the patient received initial testing for SARS-COV-2, but her polymerase chain reaction (PCR) test was negative. Additional laboratory testing was performed, and 
bilirubin was now elevated at 8.3, Alkaline Phosphatase was 117, and AST was 67 (ALT was not reported). Her gammaglutamyl transferase (GGT) was elevated at 182 IU/L (normal: 0$60)$ suggesting liver damage but not the exact cause of the injury. She was tested for acute hepatitis, but was negative for $A, B$, and $C$. The patient underwent an abdominal ultrasound one week later revealing her liver size in the upper limits of normal with a small hemangioma. Reverse hepatoportal flow was noted within the portal vein with minimal flow in the midhepatic vein. Early signs of cirrhosis were noted. Repeat labs showed decreased albumin at $3.5 \mathrm{~g} / \mathrm{dL}$ (normal: 3.9-5.0), severely elevated bilirubin (10.4), alkaline phosphatase (139), AST (80), and ALT (52). The patient was suspected to have BuddChiari Syndrome and was transferred to a large teaching hospital in a nearby city. The patient was further evaluated for portal vein thrombosis. A CT scan of the abdomen and pelvis revealed a nonocclusive thrombus in the superior mesenteric vein (SMV) and extensive portal vein thrombosis. She was given a definitive diagnosis of Budd-Chiari Syndrome with acute on chronic clot burden. The patient was started on a heparin drip and scheduled for paracentesis to relieve the ascites. Her hypercoagulability work-up revealed she was Coombs positive, indicating an immune mediated destruction of red blood cells and had a Janus Kinase-2 (JAK-2) V617F mutation in 5\% of cells.

The patient was scheduled for a transjugular intrahepatic portosystemic shunt (TIPS) procedure to reroute blood flow and decompress the congested liver, but this procedure failed. She was discharged from the hospital on the Direct Factor Xa Inhibitor apixaban $5 \mathrm{mg}$ twice daily for anticoagulation and ondansetron ODT $8 \mathrm{mg}$ three times daily for nausea. The patient was to return to the hospital for follow-up with hematology/oncology two weeks after discharge for further evaluation. However, the patient presented back to her primary care physician five days post-discharge complaining of nausea, vomiting, decreased appetite, abdominal pain, and right upper quadrant pain rated as $10 / 10$ on a numeric pain scale. She reported a score of 10 on the numeric pain scale, and her pain was rated by the provider as moderate to severe because she was able to communicate and ambulate, and no wincing or grimacing were noted outside of when the abdominal area was palpated. Ascites, and jaundiced skin and sclerae were also noted by the provider. The patient was scored as having moderate single episode depression with a score of 10 on the Patient health Questionnaire (PHQ-9). The patient's abdomen was distended with diminished bowel sounds accompanied by liver tenderness and hepatomegaly. She was counseled on her new diagnosis of BCS and treatment guidelines were searched to provide assistance in choosing short-term pain management options, yet no such guidelines exist.

The nurse practitioner consulted the collaborating clinical pharmacist for recommendations in treating pain associated with Budd-Chiari syndrome, but there were few options available to treat moderate to severe pain in this young woman with a new presentation of depression and a clotting disorder. Concerns for the clinical care team with available analgesics included providing adequate pain management without inducing dependence by using opioids, inflicting more liver damage with acetaminophen, or exacerbating her increased bleeding risk by initiating nonsteroidal antiinflammatory drugs. After consulting with pharmacy, several primary care physicians, and the hematology specialist from the discharging hospital, tramadol $25 \mathrm{mg}$ every eight hours as needed for seven days was prescribed.

\section{Discussion}

The patient's JAK-2 mutation was thought to be the underlying cause of thrombus formation leading to BCS. Although she was classified as obese with a body mass index of 32.3, no other underlying risk factors such as sickle cell disease, pregnancy, or oral contraceptive use were present. JAK-2 mutation has been associated with hypercoagulability in splanchnic and nonsplanchnic sites and is found to be an underlying condition in 29-60\% of Budd Chiari patients. ${ }^{2,4-7}$ JAK-2 gene codes for a tyrosine kinase associated with cytokine and growth factor receptors necessary for signal transduction in hematopoietic cells. A mutation in JAK-2 is usually associated with a myeloproliferative neoplasm (MPN), but this is not always the case. $^{8}$ Its significance in BCS is thought to be associated with the mutation's role in erythropoietin receptor signaling and thrombotic events though the true mechanism of action of these events and their relationship to BCS is not well known. ${ }^{2,7,9}$ This can lead to greater occlusion and thrombotic complications with BCS. $^{10}$ Despite these findings, a study analyzing clinical outcomes of BCS patients with JAK-2 mutation in China found no significant correlation between the presence of JAK-2 mutation and survival. ${ }^{11}$ While awaiting a second attempt at the TIPS procedure, she was anticoagulated with apixaban $5 \mathrm{mg}$ twice daily. Anticoagulation is the mainstay of BCS therapy to decrease thrombus formation and minimize further complications. Direct oral anticoagulants such as apixaban are more convenient for patients as they do not require INR monitoring like Vitamin $\mathrm{K}$ antagonists. They can also be used in patients with moderate hepatic impairment without dose adjustments and have a similar safety profile when used in BCS. $^{12-13}$

Individuals with BCS often present with abdominal pain which was the causal factor in the patient returning to her primary care provider after hospital discharge. A search of the literature revealed no specific pharmacological options or guidance for analgesia for Budd Chiari Syndrome. The guidelines for the management of postoperative pain suggest a multimodal approach for pain management including around the clock nonopioid analgesics and nonpharmacologic therapies. ${ }^{14}$ Nonopioid analgesics such as acetaminophen and NSAIDs are also recommended by the Centers for Disease Control (CDC) and the American Academy of Family Physicians (AAFP) for postoperative pain. ${ }^{15,16}$ Acetaminophen alone, NSAIDs such as naproxen and ibuprofen, or opioids such as hydrocodone 
(combined with acetaminophen) are the most commonly used prescription medications for moderate to severe pain. ${ }^{14-17}$ Acetaminophen, which is hepatically metabolized and could cause further liver damage/toxicity, was not considered in this patient since BCS directly affects the liver to cause acute liver injury and portal venous thrombosis. The use of NSAIDS concomitantly with direct oral anticoagulants like apixaban can increase the risk of serious bleeding. ${ }^{18}$ To minimize bleeding risk, the team decided it was best not to treat the pain with NSAIDs.

The next step in pain management was to consider an opioid, as appropriate. ${ }^{16-17}$ Opioids such as hydrocodone and oxycodone are typically combined with acetaminophen or NSAIDs in moderate to severe pain ${ }^{15}$ and were not considered due to the aforementioned reasons. In severe pain cases, sole use of full opioid agonists is considered if combined opioidnonopioid therapy is inadequate. ${ }^{16}$ Full opioid agonists such as oxycodone posed the increased risk of persistent opioid use, dependence, and abuse, particularly in a patient with depression. Tramadol is commonly used for moderate acute pain in adults in outpatient settings, is not dispensed in combination with acetaminophen or NSAIDS, and has a preferred lower abuse potential compared to other opioids. ${ }^{18}$ The adverse event profile associated with tramadol is minimal, but includes dizziness, constipation, nausea, and somnolence. Tramadol can increase seizure risk and the risk of serotonin syndrome when used with other serotonergic drugs. ${ }^{20}$ This patient was not taking any medications that would cause major interactions or increase her risk of adverse events if taken concurrently with tramadol. With this in mind, we chose to treat the patient with tramadol $25 \mathrm{mg}$ every 8 hours as needed for seven days. The pain management at this particular time was provided by a nurse practitioner. Nurse practitioners in this Southern US state cannot write prescriptions for Schedule II (narcotic) medications. This also supported our decision to prescribe tramadol. The dose for tramadol could be increased as needed and tolerated to $100 \mathrm{mg}$ every four to six hours if pain management remained insufficient. ${ }^{20}$

\section{Conclusion}

The patient reported good pain control with tramadol upon follow-up phone call. She rated her pain as 3 to 4 out of 10 on the numerical pain scale and was able to independently perform most activities of daily living while awaiting her followup appointment to the hospital. She tolerated the pain medication with no complications and underwent a successful TIPS procedure one month later. While this case ended with a positive result, it highlighted the need for additional pain management guidance for Budd-Chiari Syndrome. Currently, the clinical guidelines for this rare disorder outlines options for dissolving blood clots, improving hepatic flow, and managing ascites, however, there is little guidance on pain management in this condition, and none to assist primary care providers who may be managing patients awaiting inpatient procedures. Abdominal pain is a common symptom of Budd Chiari
Syndrome. Guidelines on treating pain associated with BCS in the outpatient setting would improve quality of life for patients waiting to undergo surgical procedures. They would also provide much needed guidance and assurance to primary care providers requiring direction on how to address the pain safely and adequately in a rare disease such as Budd Chiari Syndrome.

\section{Disclosure}

We declare no conflicts of interest or financial interests that the authors or members or their immediate families have in any product or service discussed in the manuscript, including grants (pending or received), employment, gifts, stock holdings, or options, honoraria, consultancies, expert testimony, patents, and royalties.

Acknowledgment: The authors would like to thank Letevia Herlong, FNP-C for consulting us for treatment options for this patient and providing pertinent information related to the case.

Conflicts of Interest: None

\section{References}

1. Aydinli M, Bayraktar Y. Budd-Chiari syndrome: etiology, pathogenesis and diagnosis. World J Gastroenterol. 2007;13(19):2693-2696. doi:10.3748/wjg.v13.i19.2693.

2. Hernández-Gea, Virginia, et al. Current Knowledge in Pathophysiology and Management of Budd-Chiari Syndrome and Non-Cirrhotic Non-Tumoral Splanchnic Vein Thrombosis." Journal of Hepatology. 2019;71(1): 175-199. doi:10.1016/j.jhep.2019.02.015.

3. Budd Chiari Syndrome. National Organization for Rare Disorders. 2020. Accessed November 12, 2020.

https://rarediseases.org/rare-diseases/budd-chiarisyndrome/.

4. Valla DC. Review Primary Budd-Chiari syndrome. Journal of Hepatology. 2009;50:195-203. doi:10.1016/j.jhep.2008.10.007.

5. Jackson W. Budd-Chiari Syndrome - Liver and Gallbladder Disorders - MSD Manual Consumer Version. MSD Manual Consumer Version. 2020. Accessed November 12, 2020. https://www.merckmanuals.com/home/liver-andgallbladder-disorders/blood-vessel-disorders-of-theliver/budd-chiari-syndrome.

6. De Stefano V, Fiorni A, Rossi A, et al. Incidence of the jak2 v617f Mutation among Patients with Splanchnic or Cerebral Venous Thrombosis and without Overt Chronic Myeloproliferative Disorders. Journal of Thrombosis and Haemostasis. 2007;5(4):708-714. doi:10.1111/j.15387836.2007.02424.x.

7. Velarde-Félix JS, Sanchez-Zazueta J, Gonzalez-Ibarra FP, et al. Budd-Chiari Syndrome in a Patient with JAK-2 V617F and Factor V G1691A Mutations. West Indian Med J. 2014;63(5):528-531. doi:10.7727/wimj.2013.228.

8. Test ID: JAK2V. Mayo Clinic Laboratories. 2020. Accessed December 15, 2020. https://www.mayocliniclabs.com/testcatalog/Clinical+and+Interpretive/31156. 
9. Kogan I, Chap D, Hoffman R, Axelman E, Brenner B, Nadir Y. JAK-2 V617F mutation increases heparanase procoagulant activity. Thromb Haemost. 2016;115(1):73-80. doi:10.1160/TH15-04-0320.

10. Janssen $\mathrm{HL}$, Leebeek FW. JAK2 mutation: The best diagnostic tool for myeloproliferative disease in splanchnic vein thrombosis?. Hepatology. 2006;44(6):1391-1393. doi:10.1002/hep.21489.

11. Peijin Zhang, Yanyan Zhang, Jing Zhang, et al. Association between JAK2 rs4495487 Polymorphism and Risk of BuddChiari Syndrome in China. Gastroenterology Research and Practice. 2015. https://doi.org/10.1155/2015/807865.

12. Martens $P$, Nevens F. Budd-Chiari syndrome. United European Gastroenterol J. 2015;3(6):489-500. doi:10.1177/2050640615582293

13. Chen A, Stecker E, Warden BA. Direct Oral Anticoagulant Use: A Practical Guide to Common Clinical Challenges. Journal of the American Heart Association.2020;9(13). https://doi.org/10.1161/JAHA.120.017559

14. Chou, Roger et al. "Management of Postoperative Pain: A Clinical Practice Guideline From the American Pain Society, the American Society of Regional Anesthesia and Pain Medicine, and the American Society of Anesthesiologists' Committee on Regional Anesthesia, Executive Committee, and Administrative Council." The Journal of Pain. 2016; 17(2): 131-57. doi:10.1016/j.jpain.2015.12.008.

15. Acute Pain: Postsurgical Pain. Centers for Disease Control. 2020. Accessed April 27, 2021. https://www.cdc.gov/acutepain/postsurgical-pain/index.html.

16. Blondell RD, Azadfard M, Wisniewski AM. Pharmacologic therapy for acute pain. Am Fam Physician. 2013 Jun 1;87(11):766-72. PMID: 23939498.

17. Dowell D, Haegerich TM, Chou R. CDC Guideline for Prescribing Opioids for Chronic Pain--United States, 2016. JAMA. 2016 Apr 19;315(15):1624-45. doi: 10.1001/jama.2016.1464. PMID: 26977696; PMCID: PMC6390846.

18. The United States Food and Drug Administration. 2012. ELIQUIS (apixaban) tablets for oral use. Retrieved from https://www.accessdata.fda.gov/drugsatfda_docs/label/20 12/202155s000lbl.pdf

19. Dunn KE, Bergeria CL, Huhn AS, et al. A Systematic Review of Laboratory Evidence for the Abuse Potential of Tramadol in Humans. Frontiers in Psychiatry. 2019;10(704):1-11. doi:10.3389/fpsyt.2019.00704.

20. The United States Food and Drug Administration. (2008). ULTRAM (tramadol hydrochloride) tablets. Retrieved from https://www.accessdata.fda.gov/drugsatfda_docs/label/20 09/020281s032s033lbl.pdf 